\title{
Review of Standard Tau decays from the B-factory Experiments
}

\author{
F. Salvatore ${ }^{\mathrm{a}}$, for the $B A B A R$ and Belle Collaborations

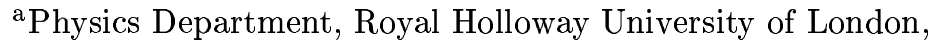 \\ Egham, Surrey, TW20 0EX, United Kingdom
}

A review of the most recent results on standard tau decays from the $B A B A R$ and Belle experiments is presented in this article.

\section{Introduction}

B-factories have been succesfully operating for more than 8 years, providing an unprecedented data sample of $e^{+} e^{-} \rightarrow$ hadrons events. The $B A B A R$ and Belle experiments have in fact already collected over $550 \mathrm{fb}^{-1}$ and $800 \mathrm{fb}^{-1}$ respectively at the $\Upsilon(4 s)$ center-of-mass (CM) energy. At this energy, the cross-section for tau production is of the same order of the cross-section for b production: $\sigma(b \bar{b}) \approx 1.1 n b \approx \sigma\left(\tau^{+} \tau^{-}\right) \approx 0.9 n b$. For this reason, B-factories can now be considered also Tau-factories.

In this paper the most recent results on standard tau decays from BABAR and Belle are presented.

\section{The BABAR and Belle Detectors}

The BABAR [1] detector operates at the PEPII asymmetric $e^{+} e^{-}$collider. Charged particles are detected and their momenta measured with a 5-layer double sided silicon vertex tracker (SVT) and a 40-layer drift chamber (DCH) inside a $1.5 \mathrm{~T}$ super-conducting solenoidal magnet. A quartz bar ring-imaging Cherenkov detector (DIRC) complements $\mathrm{d} E / \mathrm{d} x$ in the drift chambers for the identification of charged particles. Energies of neutral particles are measured by an electromagnetic calorimeter (EMC) composed of 6, $580 \mathrm{CsI}(\mathrm{Tl})$ crystals, and the instrumented magnetic flux return (IFR) is used to identify muons and $K_{L}^{0}$ mesons. The Belle [2] detector operates at the KEKB [3] asymmetric $e^{+} e^{-}$collider. The detector consists of a silicon vertex detector (SVD), a 50-layer central drift chamber (CDC), an array of aerogel threshold Cherenkov counters (ACC), a barrel-like arrangement of time-of-flight scintillation counters (TOF) and an electromagnetic calorimeter (ECL) comprised of $\mathrm{CsI}(\mathrm{Tl})$ crystals. All these detectors are located inside a super-conducting solenoidal coil that provides a $1.5 \mathrm{~T}$ magnetic field. An iron flux return outside the coil is instrumented to identify muons and $K_{L}^{0}$ mesons (KLM).

\section{Measurement of the Tau Lepton Mass}

Masses of quarks and leptons are fundamental parameters of the Standard Model (SM). The present value of the $\tau$ mass in the PDG [4], which has an accuracy of $0.3 \mathrm{MeV} / c^{2}$, is dominated by the result from the BES experiment [5]. The same level of accuracy has been reported by the KEDR Collaboration at this conference [6]. The data collected by the Belle Collaboration allow a measurement of the $\tau$ mass with similar precision [7]. A combination of all the measurement will eventually improve significantly the accuracy of the $\tau$ mass determination. Moreover, the analysis of individual $\tau$ lepton decays allows to measure the masses of positive and negative $\tau$ 's separately and therefore test the CPT theorem. This measurement cannot be performed at threshold experiments, like BES and KEDR, but can be made at the B-factories [7], and significant improvement is expected with respect to the current limit from LEP [8]. In Belle's analysis, the $\tau$ mass is determined from hadronic $\tau$ decays, using the pseudo-mass technique [9]. The estimator of the $\tau$ mass is defined by $M_{\min }=$ $\sqrt{2\left(E_{\text {beam }}-E_{X}\right)\left(E_{X}-p_{X}\right)+M_{X}^{2}}$. In absence 
of initial and final state radiation and assuming a perfect measurement of the four momentum of the hadronic system $\mathrm{X}, M_{\min }$ is distributed below the $\tau$ mass and has an edge at $M_{\tau}$. Intial and final state radiation and the finite momentum resolution of the detector smear the edge of $M_{\min }$ around $M_{\tau}$. The threshold position obtained from the fit to the experimental $M_{\min }$ distribution is used as an estimator of $M_{\tau}$. The analysis is based on $414 f b^{-1}$ of data; $\tau^{+} \tau^{-}$events are selected with one $\tau$ decaying to a well identified lepton, and the other $\tau$ decaying into $3 \pi^{ \pm} \nu_{\tau}$. The $M_{\text {min }}$ distribution of the selected data events (Figure 1) is fitted with the function: $F(x)=$ $\left(p_{3}+p_{4} * x\right) \times \operatorname{atan}\left(\left(x-p_{1}\right) / p_{2}\right)+p_{5}+p_{6} * x$ to extract a value for the mass estimator $p_{1}$. Taking into account various systematic uncertainties, the most important being the calibration of the tracking system, the choice of the fit range and shape of the threshold function and the beam energy, the final result is $M_{\tau}=1776.61 \pm 0.13$ (stat) \pm 0.35 (syst) $\mathrm{MeV} / c^{2}$, in agreement with the PDG average [4]. The pseudo-mass method allows a separate measurement of the masses of the positively and negatively charged $\tau$ leptons by doing independent fits to the two distributions. Most of the sources of systematic errors affect the result on $\tau^{+}$and $\tau^{-}$in the same way, so their contributions to the mass difference cancel. An upper limit on the relative mass difference that includes both statistic and systematic errors is obtained using the frquentist approach described in [10]: $\left|M_{\tau^{+}}-M_{\tau^{-}}\right| / M_{A v g}<2.8 \times 10^{-4}$ at $90 \% \mathrm{CL}$, one order of magnitude better than the current limit [4].

\section{Tau Decay Modes with Strange Mesons}

The $\tau$ is the only lepton with sufficiently large mass to decay to hadrons. These decays proceed via $W$ exchange and thus the decay rates to the final states containing a strange quark is suppressed by a factor $\left(\left|V_{u s}\right| /\left|V_{u d}\right|\right)^{2}$ relative to the non-strange final states, where $\left|V_{u s}\right|$ and $\left|V_{u d}\right|$ are the moduli of the Cabibbo-Kobayashi-Maskawa $(\mathrm{CKM})$ matrix $[11,12]$ elements. For a given value of the strange quark mass $m_{s}[13],\left|V_{u s}\right|$ can be determined with relatively small theoretical uncer-

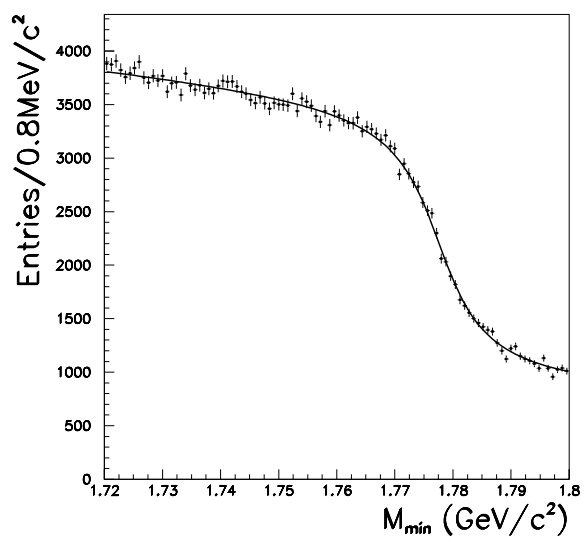

Figure 1. The pseudo-mass distribution $\left(M_{\min }\right)$ for the $\tau^{ \pm} \rightarrow 3 \pi^{ \pm} \nu_{\tau}$ candidates. The points with error bars are data and the solid line is the result of the fit with the function $F(x)$ given in the text.

tainties $[14,15]$ using the measurements of branching fractions of $\tau$ decays into final states containing an odd number of kaons.

The BABAR Collaboration has recently published a measurement of the $\tau^{-} \rightarrow K^{-} \pi^{0} \nu_{\tau}$ branching fraction [16], based on $230.2 \mathrm{fb}^{-1}$ of data containing 211.6 million $\tau$-pairs. Each event is divided in two hemispheres in the CM frame using the plane perpendicular to the thrust axis. Only those events with one charged track in each hemisphere consistent with originating from the interaction point are selected. One of these tracks is required to be identified as a lepton, while the one in the opposite hemisphere is required to be identified as a kaon. Moreover, only events containing one $\pi^{0}$ are retained. To account for the substantial energy carried away by the neutrinos, the total missing momentum in the event should exceed $0.5 \mathrm{GeV} / c$. Figure 2 shows the invariant mass spectrum of the selected $K^{-} \pi^{0}$ candidates and simulated backgrounds. The $K^{*}(892)^{-}$resonance is seen prominently above the simulated backgrounds. The branching fraction $\mathcal{B}\left(\tau^{-} \rightarrow\right.$ $\left.K^{-} \pi^{0} \nu_{\tau}\right)$ is found to be $(0.416 \pm 0.003$ (stat) \pm 0.018 (syst))\%, where the statistical uncertainty 


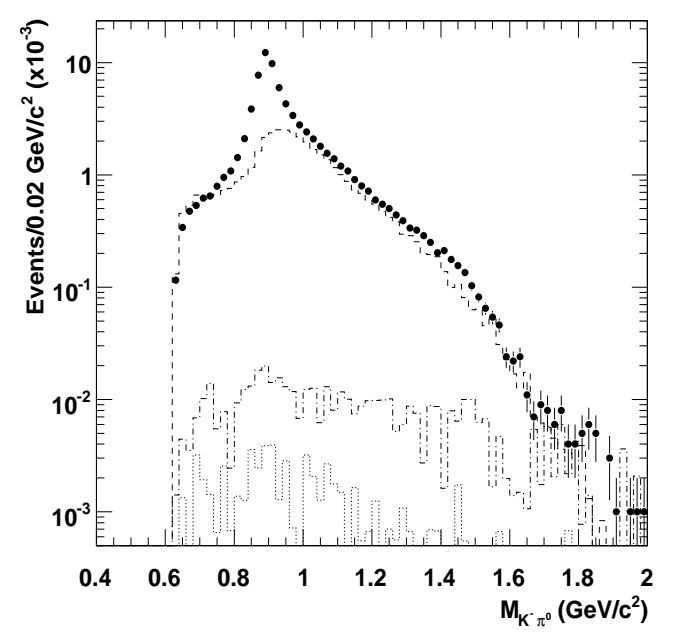

Figure 2. Distribution of the reconstructed $K^{-} \pi^{0}$ mass in the BABAR analysis. The dots are the data, while the histograms are background MC events: $\tau$ background (dashed line), $q \bar{q}$ (dashdotted line), $\mu^{+} \mu^{-}$(dotted line).

comes from the uncertainty in the number of signal events and the main systematic uncertainty comes from the efficiency of detecting a $\pi^{0}$. This is consistent with the world average and has a better accuracy.

The $\tau^{-} \rightarrow K_{s} \pi^{-} \nu_{\tau}$ branching fraction has recently been published by the Belle Collaboration [17], based on $351 \mathrm{fb}^{-1}$ of data containing 313 million $\tau$-pairs. Also in this case, events are selected in which one $\tau$ decays to leptons, while the other one decays via the hadronic channel $\tau^{-} \rightarrow h^{-} \nu_{\tau}$. Particle identification is obtained by means of ratio of likelihood functions, build for each particle hypothesis using the information on the specific particle identification detectors. Figure 3 shows the invariant mass spectrum of the selected $K_{s} \pi^{-}$candidates. The measured branching fraction $\mathcal{B}\left(\tau^{-} \rightarrow K_{S}^{0} \pi^{-} \nu_{\tau}\right)=(0.404 \pm$ 0.002 (stat) \pm 0.013 (syst)) $\%$ is consistent with the world average value and has better accuracy. An analysis of the $K_{s} \pi^{-}$invariant mass spectrum reveals contributions from the $K^{*}(892)^{-}$resonance as well as other states. To describe the enhancement near threshold a $K_{0}^{*}(800)$ amplitude is in-

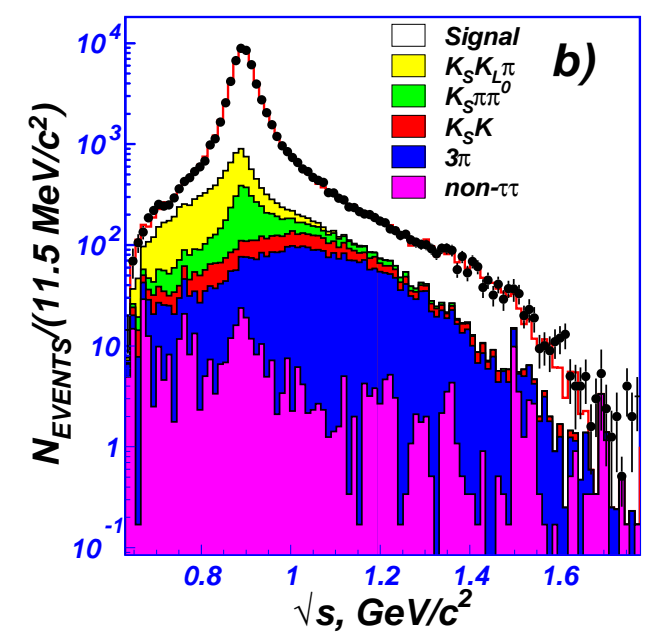

Figure 3. Distribution of the reconstructed $K_{s} \pi^{-}$ mass in the Belle analysis. The dots are the data, while the histograms are background $\mathrm{MC}$ events.

troduced. For the description of the distribution at higher invariant masses, both the $K^{*}(1410)$ vector resonance or the scalar $K_{0}^{*}(1430)$ are used. At present it is not possible to distinguish between the $K_{0}^{*}(800)+K^{*}(892)+K^{*}(1410)$ and the $K_{0}^{*}(800)+K^{*}(892)+K_{0}^{*}(1430)$ fit models. For the fist time, the $K^{*}(892)^{-}$mass and width have been measured in $\tau$ decays: $m\left(K^{*}(892)\right)=(895.47 \pm 0.20$ (stat) \pm 0.44 (syst) \pm $0.59(\bmod )) \mathrm{MeV} / c^{2}$, and $\Gamma\left(K^{*}(892)\right)=(46.2 \pm$ 0.6 (stat) \pm 1.0 (syst) \pm 0.7 (mod) $) \mathrm{MeV}$. The $K^{*}(892)^{-}$mass is significantly different from the current world average value of $(891.66 \pm$ 0.26) $\mathrm{MeV} / \mathrm{c}^{2}[4]$ and systematically lower than existing measurements listed in [4]. It has to be noted that all earlier measurements of the $K^{*}(892)^{-}$mass come from analyses of hadronic reactions and include effect of final state interaction, while in this work the decay products of the $K^{*}(892)^{-}$are the only hadrons involved. It is also worth noting that none of these previous measurements include systematic uncertainties in their results.

Using $342 \mathrm{fb}^{-1}$ of data, the BABAR Collaboration has published the exclusive branching fraction measurement of $\tau^{-} \rightarrow h^{-} h^{+} h^{-} \nu_{\tau}$, where 
each of the $h^{ \pm}$is either a pion or a kaon [18]. The signal sample is obtained by selecting events with four well reconstructed tracks having zero total charge. The event is divided in two hemispheres, one containing one charged track identified as a lepton and the other containing three charged tracks identified as coming from a $\tau^{-} \rightarrow \pi^{-} \pi^{+} \pi^{-} \nu_{\tau}, \tau^{-} \rightarrow K^{-} \pi^{+} \pi^{-} \nu_{\tau}, \tau^{-} \rightarrow$ $K^{-} K^{+} \pi^{-} \nu_{\tau}$ or $\tau^{-} \rightarrow K^{-} K^{+} K^{-} \nu_{\tau}$ decay, with decays having the wrong charge combination removed. The branching fractions obtained in this analysis are summarized in Table 1 . They are significantly more precise than the current world average results in [4]. This analysis first measures the $\tau^{-} \rightarrow K^{-} K^{+} K^{-} \nu_{\tau}$ branching fraction, with a significance in excess of $8 \sigma$. A $\phi(1020)$ contribution is seen in both the $\tau^{-} \rightarrow K^{-} K^{+} \pi^{-} \nu_{\tau}$ and $\tau^{-} \rightarrow K^{-} K^{+} K^{-} \nu_{\tau}$ decay modes (see Figure 4). The branching fractions for these two decay modes are $\mathcal{B}\left(\tau^{-} \rightarrow \phi \pi^{-} \nu_{\tau}\right)=(3.42 \pm$ $0.55 \pm 0.25) \times 10^{-5}$ and $\mathcal{B}\left(\tau^{-} \rightarrow \phi K^{-} \nu_{\tau}\right)=$ $(3.39 \pm 0.20 \pm 0.28) \times 10^{-5}$, with a significance of $5.7 \sigma$ and $9.8 \sigma$, respectively.

Using the new branching fraction measurements of $\tau^{-} \rightarrow K^{-} \pi^{0} \nu_{\tau}, \tau^{-} \rightarrow K_{s} \pi^{-} \nu_{\tau}$ and $\tau^{-} \rightarrow K^{-} \pi^{+} \pi^{-} \nu_{\tau}$ reported in this section it is possible to determine $\left|V_{u s}\right|$ from hadronic tau decays [19]. These new branching fractions are averaged with the results in [14] and the total branching fraction into strange final states is also calculated by replacing $\mathcal{B}\left(\tau^{-} \rightarrow K^{-} \nu_{\tau}\right)$ with the value obtained from theoretical predictions using the much better known $K^{-} \rightarrow \mu^{-} \nu_{\mu}(\gamma)$ decay rate and assuming $\tau-\mu$ universality: $(7.15 \pm$ $0.03) \times 10^{-3}$. The updated $\left|V_{u s}\right|$ value is $\left|V_{u s}\right|=$ $0.2157 \pm 0.0031$ using the measured $\tau$ branching fractions alone, and $\left|V_{u s}\right|=0.2171 \pm 0.0030$ using the predicted $\tau^{-} \rightarrow K^{-} \nu_{\tau}$ as well. The uncertainties are dominated by the $\sim 2 \%$ experimental errors on the $\tau$ branching fraction measurements, that have been reduced by almost a factor of two thanks to the new BABAR and Belle results.

\section{Measurement of Hadronic $\tau$ Decays in Modes with $\eta$ Mesons}

Decay modes including the $\eta$ meson(s) are interesting for testing the Wess-Zumino-Witten

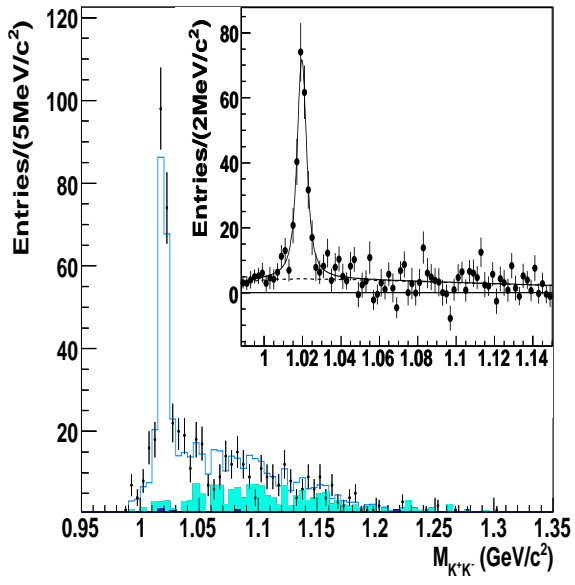

Figure 4. $K^{+} K^{-}$invariant mass distribution in $\tau^{-} \rightarrow K^{-} K^{+} K^{-} \nu_{\tau}$ decays with two entries per event. The dots are the data, the open histogram is the $\mathrm{MC}$ calculation of $\tau^{-} \rightarrow \phi K^{-} \nu_{\tau}$, the light shaded histogram is the cross feed from other $\tau^{-} \rightarrow h^{-} h^{+} h^{-} \nu_{\tau}$ channels and the dark shaded histogram represents the non- $\tau$ backgroud.

(WZW) anomaly [20,21], chiral theory [22,23] and relations to $e^{+} e^{-}$cross-sections following from conservation of the vector current (CVC) [24]. The branching fractions of $\tau^{-} \rightarrow K^{-} \eta \nu_{\tau}$, $\tau^{-} \rightarrow K^{-} \pi^{0} \eta \nu_{\tau}, \tau^{-} \rightarrow \pi^{-} \pi^{0} \eta \nu_{\tau}$ and $\tau^{-} \rightarrow$ $K^{*}(892)^{-} \eta \nu_{\tau}$ are measured by the Belle Collaboration [25], where the latter decay mode is evaluated from the corresponding $\tau^{-} \rightarrow K^{-} \pi^{0} \eta \nu_{\tau}$ measurement. A total integrated luminosity of $485 \mathrm{fb}^{-1}$ of data are used for the analysis, corresponding to 430 million $\tau$-pairs. Signal events are selected requiring that one of the $\tau$ 's decays leptonically, while the other $\tau$ decays to $X \eta \nu_{\tau}$, where $X$ denotes $K^{-}, K^{-} \pi^{0}$ or $\pi^{-} \pi^{0}$. Candidate $\eta$ mesons are reconstructed through the $\gamma \gamma$ or $\pi^{+} \pi^{-} \pi^{0}$ decay modes, while charged particles are identified using likelihood ratios based on the information obtained from the particle identification detectors. The branching fractions for the four different $\tau$ decay modes are summarized in Table 2,together with the previous measurements from CLEO [26,27] and ALEPH [28]. The result 
Table 1

Branching fraction results for the BABAR $\tau^{-} \rightarrow h^{-} h^{+} h^{-} \nu_{\tau}$ analysis.

\begin{tabular}{lll}
\hline & \multicolumn{1}{c}{$\pi^{-} \pi^{+} \pi^{-}$} & \multicolumn{1}{c}{$K^{-} \pi^{+} \pi^{-}$} \\
\hline $\mathcal{B}$ & $(88.3 \pm 0.1 \pm 1.3) \times 10^{-3}$ & $(2.73 \pm 0.02 \pm 0.09) \times 10^{-3}$ \\
$\mathcal{B}(\mathrm{PDG})$ & $(91.3 \pm 0.5 \pm 4.6) \times 10^{-3}$ & $(3.3 \pm 0.5) \times 10^{-3}$ \\
\hline & \multicolumn{1}{c}{$K^{-} K^{+} \pi^{-}$} & \multicolumn{1}{c}{$K^{-} K^{+} K^{-}$} \\
\hline $\mathcal{B}$ & $(1.346 \pm 0.010 \pm 0.036) \times 10^{-3}$ & $(1.58 \pm 0.13 \pm 0.12) \times 10^{-5}$ \\
$\mathcal{B}(\mathrm{PDG})$ & $(1.54 \pm 0.09) \times 10^{-3}$ & $<3.5 \times 10^{-5} 90 \% \mathrm{CL}$ \\
\hline
\end{tabular}

obtained in this analysis for the $\tau^{-} \rightarrow \pi^{-} \pi^{0} \eta \nu_{\tau}$ decay mode is consistent with predictions based on CVC and experimentally measured $e^{+} e^{-} \rightarrow$ $\pi^{+} \pi^{-} \eta$ cross-sections [24]. The values of the branchin fractions obtained for $\tau^{-} \rightarrow K^{-} \eta \nu_{\tau}$ and $\tau^{-} \rightarrow K^{-} \pi^{0} \eta \nu_{\tau}$ decays differ slightly from the predictions in [23].

\section{Search for the $\tau^{-} \rightarrow 3 \pi^{-} 2 \pi^{+} 2 \pi^{0} \nu_{\tau}$ decay}

Tau decays to 5 or 7 prongs have much lower branching ratios than decays in one or three charged tracks, but the unprecedented integrated luminosity reached by the B-factory experiments allows at present a detailed searches for these high multiplicity $\tau$ decay modes. The BABAR experiment has recently published an analysis on the search for the $\tau^{-} \rightarrow 3 \pi^{-} 2 \pi^{+} 2 \pi^{0} \nu_{\tau}$ decay [29] in $232 \mathrm{fb}^{-1}$ of data. This mode is highly suppressed [30,31] and therefore, if observed, it may lead to a more stringent limit on the $\tau$ neutrino mass. Events are divided in two hemispheres, where one hemisphere is required to have only one charged track, while the other hemisphere contains 5 charged tracks and exactly 2 candidate $\pi^{0} \mathrm{~s}$. The signal yield is computed in the region defined by $\left(1.3<M_{\tau}^{*}<1.8\right) \mathrm{GeV} / c^{2}$, where $M_{\tau}^{*}$ is the pseudo-mass of the $\tau$ [9]. The expected background contribution from generic $\tau$ decays is computed from $\mathrm{MC}$ events, while the $q \bar{q}$ background $(q=u, d, s, c)$ is estimated directly from the data, by subtracting the $\tau$ background contribution from the pseudo-mass distribution and fitting the resulting histogram above the signal region (see Figure 5). The fit function is then extrapolated below $1.8 \mathrm{GeV} / c^{2}$ and its integral between 1.3 and $1.8 \mathrm{GeV} / c^{2}$ yields the $q \bar{q}$ esti- mate. The total background estimate is $6.5_{-1.4}^{+2.0}$ events and a total of 10 events are observed in the data, giving therefore no evidence for the $\tau^{-} \rightarrow 3 \pi^{-} 2 \pi^{+} 2 \pi^{0} \nu_{\tau}$ decay. Figure 5 shows the final pseudo-mass spectrum in data, with superimposed the expected background PDF. The extractd upper limit for the branching fraction is $\mathcal{B}\left(\tau^{-} \rightarrow 3 \pi^{-} 2 \pi^{+} 2 \pi^{0} \nu_{\tau}\right)<3.4 \times 10^{-6}$, at $90 \%$ CL. In addition to the inclusive result, also the resonant decay mode $\tau^{-} \rightarrow 2 \omega \pi^{-} \nu_{\tau}$, with $\omega \rightarrow$ $\pi^{+} \pi^{-} \pi^{0}$ is searched for. The total number of expected background events is $0.4_{-0.4}^{+1.0}$ and one event survives all the analysis criteria. The upper limit for the branching fraction of the decay is $\mathcal{B}\left(\tau^{-} \rightarrow 2 \omega \pi^{-} \nu_{\tau}\right)<5.4 \times 10^{-7}$ at $90 \%$ CL.

\section{Acknowledgements}

I would like to thank the Tau working groups from the BABAR and Belle Collaborations for their assistance and support in the preparation of this talk.

\section{REFERENCES}

1. BABAR Collaboration, B. Aubert et al, Nucl. Inst. Meth. A 479, 1 (2002).

2. Belle Collaboration, A. Abashian et al, Nucl. Inst. Meth. A 479, 117 (2002).

3. S. Kurokawa and E. Kikutani, Nucl. Inst. Meth. A 499, 1 (2003).

4. Review of Particle Physics, W.-M. Yao et al, J. Phys. G 33, 1 (2006).

5. BES Collaboration, J. Z. Bai et al, Phys. Rev. D 53, 20 (1996).

6. KEDR Collaboration, these proceedings.

7. Belle Collaboration, K. Belous et al, arXiv:0608.046v2 [hep-ex] (2007). 
Table 2

Branching fraction results for the Belle analysis of $\tau$ decays in modes with $\eta$ meson.

\begin{tabular}{|c|c|c|}
\hline & $\overline{K^{-} \eta}$ & $\pi^{-} \pi^{0} \eta$ \\
\hline$\overline{\mathcal{B}}$ & $(1.62 \pm 0.05 \pm 0.09) \times 10^{-4}$ & $(1.39 \pm 0.03 \pm 0.07) \times 10^{-3}$ \\
\hline $\mathcal{B}($ CLEO [26]) & $(2.6 \pm 0.5 \pm 0.5) \times 10^{-4}$ & $(1.7 \pm 0.2 \pm 0.2) \times 10^{-3}$ \\
\hline $\mathcal{B}(\mathrm{ALEPH}[28])$ & $(2.9 \pm 1.3 \pm 0.7) \times 10^{-4}$ & $(1.8 \pm 0.4 \pm 0.2) \times 10^{-3}$ \\
\hline & $\overline{K^{-} \pi^{0} \eta}$ & $K^{*}-\eta$ \\
\hline$\overline{\mathcal{B}}$ & $(4.7 \pm 1.1 \pm 0.4) \times 10^{-5}$ & $(1.13 \pm 0.19 \pm 0.07) \times 10^{-4}$ \\
\hline $\mathcal{B}($ CLEO [27]) & $(17.7 \pm 5.6 \pm 7.1) \times 10^{-3}$ & $(2.90 \pm 0.80 \pm 0.42) \times 10^{-4}$ \\
\hline
\end{tabular}

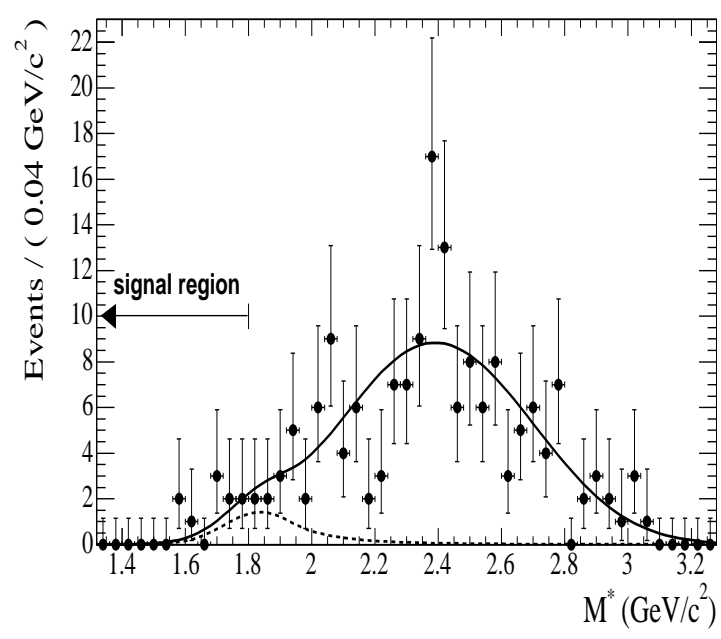

Figure 5. Pseudo-mass distribution of the data events passing the $\tau^{-} \rightarrow 3 \pi^{-} 2 \pi^{+} 2 \pi^{0} \nu_{\tau}$ selection criteria. The solid curve represents the total expected background PDF and the dashed line represents the $\tau$ background contribution.

8. OPAL Collaboration, G. Abbiendi et al, Phys. Lett. B 492, 23 (2000).

9. ARGUS Collaboration, H. Albrecht et al, Phys. Lett. B 292, 221 (1992).

10. G. Feldman and R.D. Cousins, Phys. Rev. D 57, 3873 (1998).

11. N. Cabibbo, Phys. Rev. Lett. 10, 531 (1963).

12. M. Kobayashi and T. Maskawa, Prog. Theor. Phys. 49, 652 (1973).

13. M. Jamin, J.A. Oller and A. Pich, Phys. Rev. D 74, 074009 (2006).

14. M. Davier, A. Höcker and Z. Zhang, Rev.
Mod. Phys. 78, 1043 (2006).

15. E. Gamiz, M. Jamin, A. Pich, J. Prades and F. Schwab, Nucl. Phys. Proc. Suppl. 169, 85 (2007).

16. BABAR Collaboration, B. Aubert et al, Phys. Rev. D 76, 051104(R) (2007)

17. Belle Collaboration, D. Epifanov et al, Phys. Lett. B 654, 65 (2007).

18. BABAR Collaboration, B. Aubert et al, Phys. Rev. Lett. 100, 011801 (2008).

19. S. Banerjee, arXiv:0707.3058v4 [hep-ex] (2007).

20. J. Weiss, B. Zumino, Phys. Lett. B 37, 95 (1971).

21. E. Witten, Nucl. Phys. B 223, 422 (1983).

22. A. Pich, Phys. Lett. B 196, 561 (1987).

23. B.A. Li, Phys. Rev. D 55, 1436 (1997).

24. S. Eidelman and V. Ivanchenko, Phys. Lett. B 257, 437 (1991).

25. Belle Collaboration, K. Abe et al, arXiv:0708.0733v1 [hep/ex] (2007).

26. CLEO Collaboration, M. Artuso et al, Phys. Rev. Lett. 69, 3278 (1992); CLEO Collaboration, J. Bartelt et al, Phys. Rev. Lett. 76, 4119 (1996).

27. CLEO Collaboration, M. Bishai et al, Phys. Rev. Lett. 82, 281 (1999);

28. ALEPH Collaboration, D. Busulic et al, Z. Phys. C 74, 263 (1997).

29. BABAR Collaboration, B. Aubert et al, Phys. Rev. D 73, 112003 (2006).

30. S. Nussinov and M.V. Purohit, Phys. Rev. D 65, 034018 (2002).

31. BABAR Collaboration, B. Aubert et al, Phys. Rev. D 72, 012003 (2005). 